\title{
A Safety Signal's Significance with the COVID-19 Coronavirus
}

\author{
Thomas W Miller* \\ Professor Emeritus \& Senior Research Scientist, Institute for Health, Intervention \& Policy, University of Connecticut, Department of Gerontology, Col- \\ lege of Public Health, \& Department of Psychiatry, College Medicine, University of Kentucky, USA
}

${ }^{\star}$ Corresponding author: Thomas W Miller, Department of Gerontology, College of Public Health, \& Department of Psychiatry, College Medicine, University of Kentucky, USA

Received: December 23, 2020; Accepted: December 29, 2020; Published: December 31, 2020

\section{Introduction}

The global pandemic involving COVID-19 (coronavirus) has produced unprecedented challenges for the medical, healthcare providers and our world community. The World Health Organization (WHO 2020) initially declared COVID-19 a pandemic, pointing to the over numerous cases of the coronavirus illness in over a hundred countries and territories around the world and the sustained risk of further global spread $[1,2]$. The term pandemic is most often applied to new influenza strains, and the Centers for Disease Control and Prevention (CDC) use it to refer to strains of virus that are able to infect people easily and spread from person to person in an efficient and sustained manner. Such a declaration refers to the spread of a disease, rather than the severity of the illness it causes. A pandemic declaration can result in increased levels of stress, anxiety, panic and levels of functional depression for some individuals [3]. Recognized is the realization that these unusual circumstances create significant uncertainty and unease in the professional and personal lives of health care professionals and their patients.

\section{Definition of a Safety Signal}

"Safety signals" are learned cues that predict the nonoccurrence of an aversive event. As such, safety signals are potent inhibitors of fear and stress responses. Investigations of safety signal learning have increased over the last few years due in part to the finding that traumatized persons are unable to use safety cues to inhibit fear, making it a clinically relevant phenotype.

The coronavirus has traumatized some which has been recognized as a state of heightened fear or anxiety in environments globally. This symptom has been conceptualized as a generalization of the fear conditioned during the traumatic experience that becomes resistant to extinction. As opposed to danger learning where a cue is paired with aversive stimulation, safety learning involves associating distinct environmental stimuli also known as safety signals that can be used an applied when aversive events occur as in a global pandemic.

During periods of high stress such as during this Covid-19 pandemic, fear often permeates the lives of many because if the unknown nature of this illness. This occurs because of the absence of a learned safety signal. Such safety signals can inhibit fear responses to cues in the environment. As such, safety signals are only learned when the subject expects danger but it does not necessarily occur. More fundamental to the clinical importance of a safety signal is the distinction between safe and dangerous circumstances. Thus, identifying the mechanisms of safety learning represents a significant goal for basic neuroscience that should inform future prevention and treatment of trauma and other anxiety disorders.

With COVID-19 global pandemic, the World Health Organization (2020) continues to ask countries to "take urgent and aggressive action." World leaders continue holding international teleconferences with health officials to address the most effective way to protect the public and develop public health policy for the coronavirus that has caused multiple illnesses and deaths worldwide.

\section{Transitioning the Pandemic}

The urgency has created stressful life experiences for all ages that pose the potential for illness resulting for some in disabling fear, a hallmark of anxiety and stress-related disorders [4]. Researchers at Yale University and Weill Cornell Medicine report on a novel way that could help combat such anxiety experienced at times like these. When life events as the spread of the Corvid 19 triggers excessive fear and the absence of a safety signal. In humans, a symbol or a sound that is never associated with adverse events can relieve anxiety through an entirely different brain network than that activated by fear and worry. Each individual must find their own "safety signal" whether that is a mantra, song, a person, or even an item like a stuffed animal that represents the presence of safety and security.

The Centers for Disease Control and Prevention (CDC), the World Health Organization (WHO), and other reputable agencies have advocated on how to address the coronavirus by washing hands frequently, avoid sharing personal items, and maintaining social distance from others beyond immediate family.

While it's still unclear exactly how much of the current coronavirus outbreak has been fueled by asymptomatic, mildly symptomatic, or pre-symptomatic individuals, the risk of contagion exists. A yet to be published article in the CDC journal "Emerging Infectious Disease" (CDC 2020) reports that the time between cases in a chain of transmission is less than a week, with more than $10 \%$ of patients being infected by someone who has the virus but does not yet have symptoms according to Dr. Luren Meyers, a professor of integrative 
biology at UT Austin, who was part of a team of scientists from the United States, France, China and Hong Kong examining this viral threat.

Earlier this year, researchers in China published a research letter in the Journal of the American Medical Association, outlining a case of an asymptomatic woman in Wuhan, China who reportedly spread the virus to five family members while traveling to Anyang, China-all of whom developed COVID-19 pneumonia. The sequence of events suggests that the coronavirus may have been transmitted by the asymptomatic carrier," [5].

\section{Prevention Interventions}

Coordinated regional efforts are underway under the direction of the Centers for Disease Control and Prevention (CDC) that provides guidelines aimed at prevention intervention. Each individual should make the effort to create one's own "safety signal" by following the recommendations of the CDC (2020). Know how it spreads and that there is currently no vaccine to prevent coronavirus disease (COVID-19). Critical for prevention is avoided exposing the virus. The virus is thought to spread mainly from person-to-person. Between people who are in close contact with one another. Through respiratory droplets produced when an infected person coughs or sneezes. These droplets can land in the mouths or noses of people who are nearby or possibly be inhaled into the lungs.

Disinfecting by washing hands often with soap and water for at least twenty seconds especially after you have been in a public place or after blowing your nose, coughing, or sneezing. If soap and water are not readily available, use a hand sanitizer that contains at least $60 \%$ alcohol. Cover all surfaces of your hands and rub them together until they feel dry. Avoid touching the eyes, nose, and mouth with unwashed hands Put distance between yourself and other people if COVID-19 is spreading in your community. This is especially important for people who are at higher risk of getting immune compromised illness.

Health care calls for "sheltering in place" are effort to provide primary prevention it's important to stay home to slow the spread of COVID-19, and if you must go out, practice personal quarantine. While we stay home, don't let fear and anxiety about the COVID-19 pandemic become overwhelming. Managing mental health issues can be aided by taking breaks from watching, reading, or listening to news stories and social media. It remains important to take the time to connect with others. Networking with friends and loved ones over the phone or via video chat about the thoughts and feelings experienced during this pandemic is very important to maintain mental health daring three times. Employ the use mindful meditation, eating healthy meals, exercising regularly, and getting plenty of sleep.
Take steps to protect yourself and others. Stay sheltered in place especially when you're sick. Shelter in place means to seek safety within the building one already occupies, rather than to evacuate the area or seek a community emergency shelter. The American Red Cross says the warning is issued when "chemical, biological, or radiological contaminants which would include exposure to the coronavirus.

Efforts must be made to cover one's mouth and nose with a tissue when you cough or sneeze or use the inside of your elbow. Throw used tissues in the trash. Immediately wash your hands with soap and water for at least 20 seconds. If soap and water are not readily available, clean your hands with a hand sanitizer that contains at least $60 \%$ alcohol.

It is important to wear a facemask for your own health as well as the health of others. Everyone should wear a facemask when they are around other people (e.g., sharing a room or vehicle) and before entering a healthcare provider's office. If someone is not able to wear a facemask due to breathing difficulties, then these individuals should cover all coughs and sneezes, and people who are caring for theme should wear a facemask when they enter ones room. Wear a facemask when caring for someone who is showing any signs or symptoms of respiratory infection and fever.

When considering the anxiety and apprehension individuals may experience with the vulnerabilities of the present pandemic and future epidemics of this proportion, patient medical education can provide a buffer against the Prevention interventions that include cleaning and disinfecting objects and surfaces that are touched regularly. This includes tables, doorknobs, light switches, countertops, handles, desks, phones, keyboards, toilets, faucets, and sinks. If surfaces are dirty, clean them: Use detergent or soap and water prior to disinfection. With first signs of symptoms, take advantage of Virtual Care in an effort to minimize unnecessary visits to an emergency room or health care provider's office, which can also decrease the spread of illness and/or infection of many conditions, including COVID-19. Finally, each individual is encouraged to establish one's own "safety signal" by adhering to the multiple precautions that include the guidelines developed and promoted by the World Health organization and the Centers for Disease Control and Prevention (CDC 2020).

\section{References}

1. Centers for Disease Control (2020) Coronavirus Disease 2019 (COVID-19).

2. World Health Organization (2020) Coronavirus disease 2019 (COVID-19): Situation Report-38.

3. Miller TW (2015) Problem Epidemics in Recent Times. Health \& Wellness. Lexington Kentucky: Rock point Publisher Incorporated.

4. Miller TW (2010) Handbook of Stressful Transitions across the Life Span. New York: Springer Publishers Incorporated

5. Huang C, Wang Y, Li X, et al. (2020) Clinical features of patients infected with 2019 novel coronavirus in Wuhan, China. Lancet 395: 497-506.

\section{Citation:}

